Cochrane Database of Systematic Reviews

\title{
Canadian C-spine rule and the National Emergency X- Radiography Utilization Study (NEXUS) for detecting clinically important cervical spine injury following blunt trauma (Protocol)
}

Saragiotto BT, Maher CG, Lin CWC, Verhagen AP, Goergen S, Michaleff ZA

Saragiotto BT, Maher CG, Lin CWC, Verhagen AP, Goergen S, Michaleff ZA.

Canadian C-spine rule and the National Emergency X-Radiography Utilization Study (NEXUS) for detecting clinically important cervical spine injury following blunt trauma.

Cochrane Database of Systematic Reviews 2018, Issue 4. Art. No.: CD012989.

DOI: $10.1002 / 14651858 . C D 012989$.

www.cochranelibrary.com

Canadian C-spine rule and the National Emergency X-Radiography Utilization Study (NEXUS) for detecting clinically important cervical spine injury following blunt trauma (Protocol)

Copyright @ 2018 The Cochrane Collaboration. Published by John Wiley \& Sons, Ltd. 


\section{TABLE OF CONTENTS}

HEADER . . . . . . . . . . . . . . . . . . . . . . . . . . . . . . . . . . . . . . 1

ABSTRACT . . . . . . . . . . . . . . . . . . . . . . . . . . . . . . . . . . . . . . 1

BACKGROUND . . . . . . . . . . . . . . . . . . . . . . . . . . . . . . . . . . 1

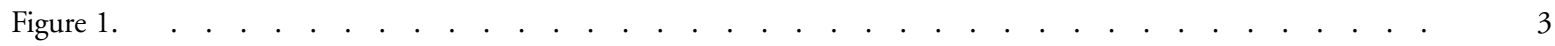

OBJECTIVES . . . . . . . . . . . . . . . . . . . . . . . . . . . . . . . . . . . . . . . . 5

METHODS . . . . . . . . . . . . . . . . . . . . . . . . . . . . . . . . . . . . . . 5

REFERENCES . . . . . . . . . . . . . . . . . . . . . . . . . . . . . . . . . . . . . . . .

ADDITIONAL TABLES . . . . . . . . . . . . . . . . . . . . . . . . . . . . . . . . . . . . . . . . . . . . . .

APPENDICES . . . . . . . . . . . . . . . . . . . . . . . . . . . . . . . . . . . . . . . .

WHAT'S NEW . . . . . . . . . . . . . . . . . . . . . . . . . . . . . . . . . . . . . . 11

HISTORY . . . . . . . . . . . . . . . . . . . . . . . . . . . . . . . . . . . . . . . 11

CONTRIBUTIONS OF AUTHORS . . . . . . . . . . . . . . . . . . . . . . . . . . . . . . . . . . . . . . . .

DECLARATIONS OF INTEREST . . . . . . . . . . . . . . . . . . . . . . . . . . . . . . . . . . . . . . . . .

SOURCES OF SUPPORT . . . . . . . . . . . . . . . . . . . . . . . . . . . . . . . . . . . . . . . . . . . . . .

Canadian C-spine rule and the National Emergency X-Radiography Utilization Study (NEXUS) for detecting clinically important 
[Diagnostic Test Accuracy Protocol]

\title{
Canadian C-spine rule and the National Emergency $X$ - Radiography Utilization Study (NEXUS) for detecting clinically important cervical spine injury following blunt trauma
}

\author{
Bruno T Saragiotto ${ }^{1}$, Christopher G Maher ${ }^{2}$, Chung-Wei Christine Lin $^{3}$, Arianne P Verhagen ${ }^{4}$, Stacy Goergen ${ }^{5}$, Zoe A Michaleff ${ }^{6}$ \\ ${ }^{1}$ Musculoskeletal Health Sydney, School of Public Health, Sydney Medical School, The University of Sydney, Sydney, Australia. \\ ${ }^{2}$ Sydney School of Public Health, The University of Sydney, Sydney, Australia. ${ }^{3}$ Musculoskeletal Health Sydney, Sydney School of \\ Public Health, The University of Sydney, Sydney, Australia. ${ }^{4}$ Department of Physiotherapy, University of Technology Sydney, Sydney, \\ Australia. ${ }^{5}$ Diagnostic Imaging, Monash Medical Centre, Southern Health, Melbourne, Australia. ${ }^{6}$ Arthritis Research UK Primary \\ Care Centre, Research Institute for Primary Care \& Health Sciences, Keele University, Keele, UK
}

Contact address: Bruno T Saragiotto, Musculoskeletal Health Sydney, School of Public Health, Sydney Medical School, The University of Sydney, Sydney, Australia. bruno.saragiotto@gmail.com.

Editorial group: Cochrane Musculoskeletal Group.

Publication status and date: Edited (no change to conclusions), published in Issue 5, 2018.

Citation: Saragiotto BT, Maher CG, Lin CWC, Verhagen AP, Goergen S, Michaleff ZA. Canadian C-spine rule and the National Emergency X-Radiography Utilization Study (NEXUS) for detecting clinically important cervical spine injury following blunt trauma. Cochrane Database of Systematic Reviews 2018, Issue 4. Art. No.: CD012989. DOI: 10.1002/14651858.CD012989.

Copyright (C) 2018 The Cochrane Collaboration. Published by John Wiley \& Sons, Ltd.

\begin{abstract}
A B S T R A C T
This is a protocol for a Cochrane Review (Diagnostic test accuracy). The objectives are as follows:

To describe and compare the diagnostic accuracy of the Canadian C-spine rule and the National Emergency X-Radiography Utilization Study (NEXUS) to screen for clinically important cervical spine injury (CSI) in patients following blunt trauma.
\end{abstract}

\section{B A C K G R O U N D}

Cervical spine injury (CSI) represents approximately $3.5 \%$ of the cases of trauma presenting to emergency departments around the world (Hasler 2012; Milby 2008; Niska 2010). Approximately $2 \%$ of cervical spine injuries will be clinically important injuries such as fracture or dislocation and require specialist intervention (e.g. immobilisation or surgical intervention). Due to the potentially catastrophic consequences of a delayed or missed diagnosis of clinically important CSI (Davis 1993), diagnostic imaging is undertaken for the great majority of patients with CSI. As the prevalence of clinically important CSI is only $2 \%$ of total CSI, a mandatory imaging policy would lead to the large majority of patients with CSI receiving imaging that confers no net health benefit (Stiell 1997).

Clinical decision rules can assist clinicians to rule out clinically important CSI by identifying those patients with a lower likelihood of a clinically important CSI and therefore do not require imaging (Motor Accidents Authority 2014; TRACsa 2008). The use of validated tools to improve clinical assessment of pre-test risk has the potential to minimise costs, resource utilisation, length of stay in emergency departments, and unnecessary exposure to radiation (Griffith 2011). Use of such tools can facilitate shared decision 
making with patients and provide them with reassurance about the reasoning behind a clinical decision to defer or not perform imaging at all.

The National Emergency X-Radiography Utilization Study (NEXUS) criteria and the Canadian C-spine rule are two clinical decision rules developed to help clinicians risk-stratify patients with cervical spine trauma to determine if they need imaging to rule out clinically important CSI. These clinical decision rules have been externally validated internationally and their use in routine clinical practice is recommended by international guidelines (Hoffman 2000; NICE Guidance 2007; The College of Emergency Medicine 2010; TRACsa 2008; Stiell 2001). For safe and effective screening, the rule must have a high-sensitivity rate, indicating that a clinically important CSI will not be missed. Other attributes of a high-performing clinical decision rule are selection criteria that allow its application to a broad range of patients at risk of the target condition and unambiguous definition/description of the items within the rule. These attributes work together to increase the likelihood of the correct use of the rule for the largest number of patients in a variety of clinical settings.

Despite evidence indicating higher sensitivity and specificity of the Canadian C-spine rule than NEXUS for patients meeting the inclusion/exclusion criteria for either of these rules (Michaleff 2012; Stiell 2003), the current imaging and other clinical practice guidelines do not reflect the differences in both performance as well as patient selection criteria for the two clinical decision rules (ACEM / RANZCR Guidelines 2012; ACR Appropriateness Criteria; Diagnostic Imaging Pathways).

\section{Target condition being diagnosed}

Clinically important CSI is defined as fracture, dislocation or mechanical instability of the cervical spine which requires specialist intervention (e.g. immobilisation or surgical intervention) (Stiell 1999; Stiell 2001). Blunt trauma results from an impact to the body in the absence of any penetrating trauma, examples of blunt trauma include motor vehicle accident, fall or assault.

The prevalence of CSI in trauma patients is estimated as less than $4 \%$, of which only about half of these will have a fracture or dislocation, of which about $25 \%$ will report neurological deficits (Hasler 2012; Milby 2008; Niska 2010). Clinically important CSI requires specific treatment (e.g. operative stabilisation) to prevent secondary injury to the spinal cord, which would lead to significant disability, morbidity or mortality (Davis 1993; Goergen 2015).

\section{Index test(s)}

The NEXUS criteria (Hoffman 1998) (Table 1) and the Canadian C-spine rule (Stiell 2001) (Figure 1) are two clinical decision rules designed to be used for patients with CSI following blunt trauma. Both decision rules were developed for patients with cervical trauma in whom clinically important CSI is a concern; however the Canadian C-spine rule specifies its use for patients who are aged 16 years or older, alert (as indicated by a score of 15 on the Glasgow Coma Scale), and in a stable condition (Stiell 2001), whereas NEXUS has one inclusion criterion (suspected clinically important CSI following trauma) and one exclusion criterion (penetrating trauma) (Hoffman 2000). For both rules, patients with a negative result have an acceptably low risk of clinically important CSI and imaging is not required. A positive test result is interpreted quite differently as both rules have only modest specificity $(<50 \%)$, meaning that most positive test results are false positives (Michaleff 2012). This means that many patients who test positive and are imaged will be subsequently shown not to have clinically important CSI. 
Figure I. The Canadian C-Spine Rule.

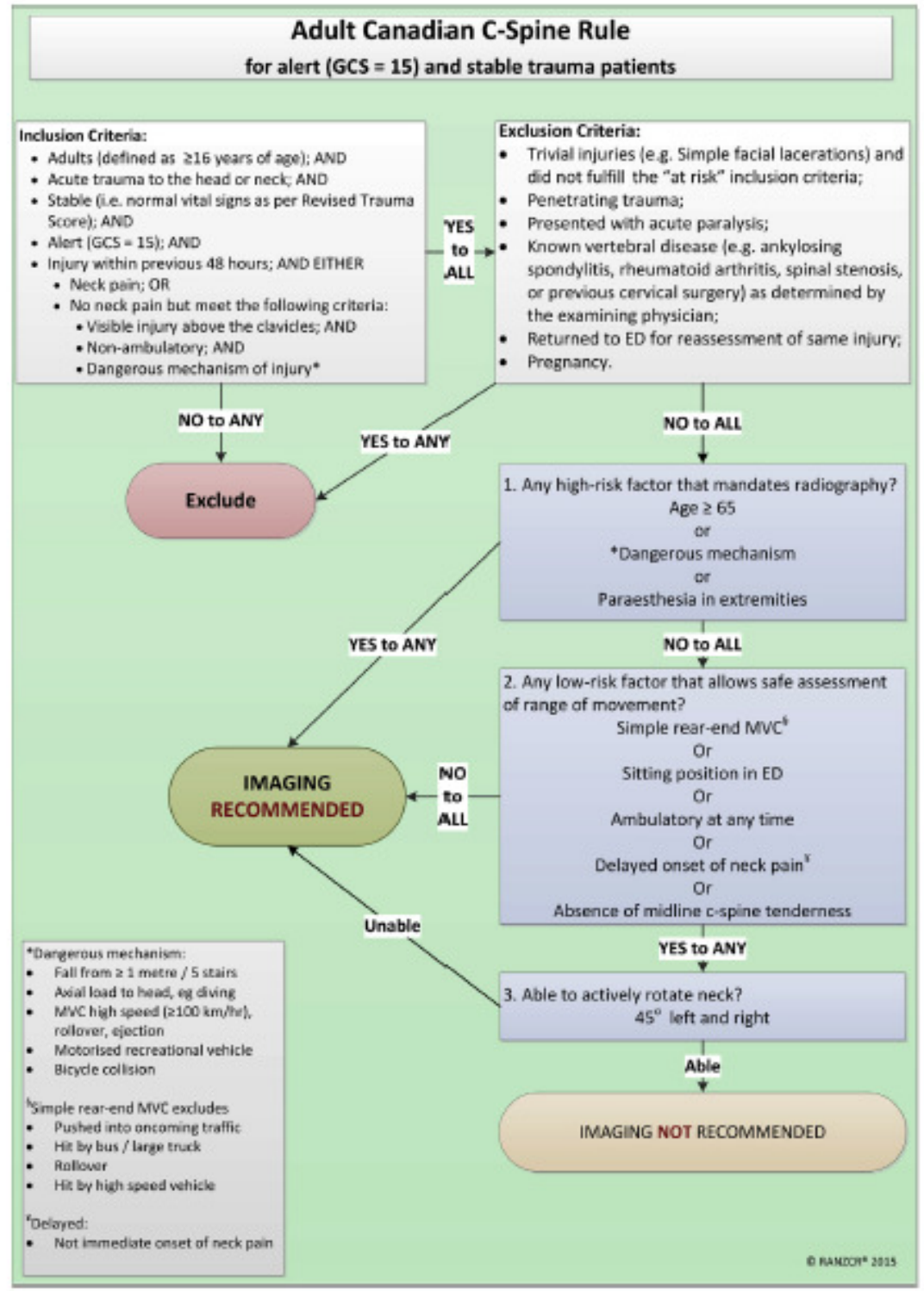

Fig 1. An example of a static PDF version of a decision support flowchart, illustrating the Canadian Adult C-Spine Rule (adult cervical spine trauma). $E D=$ emergency department; $M V C=$ motor vehicle collision. 
The Canadian C-spine rule consists of a) three high-risk criteria, the presence of any one of which results in a recommendation for imaging (age $\geq 65$ years, dangerous mechanism, or paraesthesia in extremities); b) five low-risk criteria the presence of any one of which allows the collar to be removed and cervical spine rotation assessed (simple rear-end motor vehicle collision, sitting position in the emergency department, ambulatory at any time, delayed onset of neck pain, or absence of midline cervical-spine tenderness); and c) an assessment of the ability of patients to rotate the neck 45 degrees left and right (Stiell 2003) (Figure 1). The five criteria of the NEXUS are: 1) no posterior midline cervical tenderness; 2) no evidence of intoxication; 3) normal level of alertness (i.e. the patient is alert and oriented to person, place, time, and event); 4) no focal neurological deficit; and 5) no painful distracting injuries (e.g. long-bone fracture) (Hoffman 1998; Hoffman 2000) (Table 1). Patients who meet all five of the NEXUS criteria can have safely clinically important CSI excluded without the use of imaging. A positive test rest for either rule suggests that the patient is more likely to have a clinically important CSI and in these patients recommends the use of imaging investigations. Neither rule however specifies the type of imaging investigation that should be used (i.e. computed tomography (CT) or plain radiography) and both are silent on whether plain radiography should be followed by CT if the patient is deemed, by some criterion (e.g. neurological abnormality or injury mechanism) to be at high risk.

\section{Clinical pathway}

When patients with CSI arrive at the emergency department, they typically undergo a series of assessments to rule out clinically important CSI. Firstly, the initial assessment includes the Glasgow Coma Scale score, history taking, which consists of medical history (i.e. history of spine surgery, previous neck pain), demographic details, mechanism of injury, presence, onset and progression of symptoms (i.e. pain, paraesthesia) (Ackland 2012; Goergen 2015). Then, examination of physical and neurological status is performed, which includes motor function (i.e. tone, strength, reflexes) and sensation (i.e. paraesthesia) (Ackland 2012; Goergen 2015). Further assessments would include the presence of bony tenderness in the midline, and the patient's ability to rotate the cervical spine 45 degrees to each side (Goergen 2015). Finally, most patients undergo imaging, usually radiography or CT (Como 2009; Daffner 2007). A semi-rigid cervical collar is often recommended until imaging can be conducted (Ackland 2012).

In practice, the choice of using CT versus plain radiography for the diagnosis of cervical spine injury is subjective and depends on many factors, such as hospital policies and protocol, the availability of imaging equipment e.g. CT, the severity of the trauma and age of the patient. In children, radiography tends to be performed first and only when it is abnormal is CT considered (Daffner 2007), and magnetic resonance imaging (MRI) may be performed instead of CT in certain situations (e.g. persistent neck pain with normal plain radiography and no neurological signs or symptoms) because of suspicion of ligamentous injury, although there is no evidence to support the clinical utility of MRI in this situation as demonstration of ligamentous oedema or even disruption does not necessarily change planned treatment (collar immobilisation). In adults who attend emergency departments with what is thought to be clinically important cervical spine trauma (this is mostly based on clinical suspicion without consistent application or understanding of decision rules), CT is usually performed first as it is more sensitive and specific than plain radiography in the demonstration of fractures and subluxation or dislocation (Parizel 2010). When CT is not available or when pre-test suspicion is low some people will carry out plain radiography despite its inferior performance (Holmes 2005). The original NEXUS and Canadian C-spine rule studies did not indicate what proportion of patients had CT versus radiography, nor did they mandate CT if imaging was indicated. However, there is evidence that supports CT being more accurate than plain radiography; plain radiography often fails to show the cervicothoracic junction adequately (Holmes 2005; Parizel 2010). Appropriate application of evidence-based clinical decision rules for patients with CSI can help to focus clinical examination and history taking, improve rates of positive imaging, and reduce unnecessary use of imaging that carries with it financial costs for the health system and opportunity costs for other patients who need to access imaging in resource-constrained environments such as public emergency departments, especially at night and on weekends.

\section{Rationale}

Clinically important CSI following blunt trauma accounts for approximately $2 \%$ of the cases of cervical spine injury in emergency departments; however, most patients undergo diagnostic imaging (Stiell 1997). The NEXUS criteria and the Canadian C-spine rule are two clinical decision rules available to assist emergency clinicians to evaluate the need for imaging in patients with CSI. These rules have the potential to rule out a clinically important cervical spine injuries and therefore reduce the number of unnecessary imaging in these patients. A review conducted in 2012 (Michaleff 2012) concluded that the Canadian C-spine rule has better diagnostic accuracy than the NEXUS criteria; however we are aware of new studies published since the search date of this review (Goode 2014; Griffith 2013; Griffith 2014; Matteucci 2015; Morrison 2014). Therefore, this new Cochrane review will be an update of the previous systematic review (Michaleff 2012).

Canadian C-spine rule and the National Emergency X-Radiography Utilization Study (NEXUS) for detecting clinically important 


\section{O B J E C T I V E S}

To describe and compare the diagnostic accuracy of the Canadian C-spine rule and the National Emergency X-Radiography Utilization Study (NEXUS) to screen for clinically important cervical spine injury (CSI) in patients following blunt trauma.

\section{METHODS}

\section{Criteria for considering studies for this review}

\section{Types of studies}

We will consider prospective cohort or cross-sectional studies if they compare the results of the Canadian C-spine rule or NEXUS with an appropriate reference standard. Only studies that enrolled clinical populations where there is diagnostic uncertainty will be included in the review. We will only include results from full-text reports that include sufficient raw data to allow reconstruction of contingency tables. If studies have been published as an abstract or conference proceeding, full-text publications will be sought, or alternatively we will contact authors for their data where possible. We will also contact the authors for data in case we are not able to construct the $2 \times 2$ tables from the reports. Studies published in languages other than English will be included if translations can be obtained.

\section{Participants}

We will include studies that assess the diagnostic accuracy of the Canadian C-spine rule or NEXUS in adults presenting with cervical spine injury after blunt trauma. Clinically important CSI is defined as fracture, dislocation or mechanical instability of the cervical spine which requires specialist intervention (e.g. immobilisation or surgical intervention) (Stiell 1999; Stiell 2001). Blunt trauma results from an impact to the body in the absence of any penetrating trauma; examples of blunt trauma include motor vehicle accident, fall or assault.

We will include studies carried out in all settings (i.e. hospital emergency departments and general practice) and will include studies in which medically trained and qualified individuals as well as nurse practitioners and allied health professionals have undertaken assessment of participants using either of the two clinical decision rules).

\section{Index tests}

We will include studies that evaluated the diagnostic accuracy of either the Canadian C-spine rule (Stiell 2001) or NEXUS rule (Hoffman 1998), or both to rule out a potentially serious CSI. Both instruments are clinical decision rules commonly used to decide whether or not diagnostic imaging is needed to prove or rule out CSI after blunt trauma. Since they have slightly different inclusion criteria (i.e. the C-spine rule is not applicable for those with Glasgow Coma Scale less than 15 and NEXUS recommends imaging for those patients), we will assess the diagnostic accuracy of each of the rules when applied in accordance with their derivation.

\section{Target conditions}

Clinically important cervical spinal injury (CSI) after blunt trauma.

\section{Reference standards}

We will include studies if the diagnostic rule results were compared to results of diagnostic imaging procedures such as plain radiographs, and computed tomography (CT) to confirm the presence of cervical spine fracture, dislocation or mechanical instability. Imaging is regarded as a valid reference standard. For the development of both tools, plain radiography was used as a reference standard, unless CT or magnetic resonance (MRI) was performed (Hoffman 2000; Stiell 2001).

We will also include studies that image some patients and clinically followed the remainder as a reference standard but will conduct a sensitivity analysis (if possible) to investigate the effect of the use of this imperfect reference standard on review results. An example of this approach is the 14-day proxy method, where the assessing clinician elects to image patients based upon their clinical judgment and the remaining patients are contacted by a registered nurse 14 days after discharge and asked questions about pain and return to function (Vandemheen 1999). A positive response to these questions results in patients being asked to return to hospital for imaging investigations.

\section{Search methods for identification of studies}

\section{Electronic searches}

We will search the following databases from their inception.

- MEDLINE (OvidSP)

- Embase (OvidSP)

- Cumulative Index to Nursing and Allied Health Literature (CINAHL) (EBSCO)

- Latin American and Caribbean Health Sciences Literature ( LILACS) (Bireme)

The search strategy for the MEDLINE database is presented on Appendix 1. We will also search ClinicalTrials.gov and the World Health Organization's International Clinical Trials Registry Platform (WHO ICTRP) for trials registry and protocols. 


\section{Searching other resources}

We will also search the reference lists of included studies and previous relevant reviews for potentially relevant studies.

\section{Data collection and analysis}

\section{Selection of studies}

Two review authors will independently screen titles and abstracts from the search results. The full-text publications of the potentially eligible studies will be retrieved and independently assessed for inclusion. Disagreements will be resolved by consensus or arbitration by a third review author. No language restrictions will be applied and translations will be sought where possible.

\section{Data extraction and management}

Two review authors will independently perform the data extraction. Disagreements will be resolved by consensus or arbitration by a third review author when required. We will extract the following data: characteristics of studies (country, recruitment modality, source of funding, risk of bias), study design, characteristics of participants (age, gender, duration of symptoms, number of participants, including number receiving the index test and reference standard), type of index tests and reference standards, including the methods of execution; experience, expertise, and training of the assessors, and the frequency of true positives, true negatives, false positives and false negatives for the index to the reference test in order to create a $2 \times 2$ table for each included study. We will contact the authors in case of incomplete or missing data.

\section{Assessment of methodological quality}

The methodological quality of included studies will be assessed using a modified version of the Quality Assessment of Diagnostic Accuracy Studies tool (QUADAS-2) (Whiting 2011; Slaar 2017; Wade 2013). The QUADAS-2 tool assesses methodological quality based on four domains: (1) patient selection, (2) index test, (3) reference standard, and (4) flow and timing. The developers of QUADAS-2 recommend that the tool can be tailored for each specific review by adding or omitting signalling questions to assist judgements. The signalling questions related to risk of bias and applicability that will be used in this review are described in Table 2. Studies judged as 'no' or 'unclear' for one or more domains are considered as having concerns regarding applicability (Whiting
2011). Two review authors will independently assess the methodological quality of the included studies. Disagreements will be resolved by discussion and, if necessary, arbitrated by a third review author.

\section{Statistical analysis and data synthesis}

We will generate diagnostic $2 \times 2$ contingency tables to record true positives, true negatives, false positives and false negatives for each study, and calculate sensitivity, specificity and the $95 \%$ confidence interval (CI) for each index test. To estimate the summary sensitivity and specificity we will perform a meta-analysis using the bivariate logistic model (Reitsma 2005). We expect that all studies share the same criteria for test positivity for each of the two tests (positivity is defined with the same threshold across studies); thus no issues of multiple thresholds reported are expected to rise. For studies that directly compared both tests, we will perform direct comparison, but comparison will not be limited to direct comparisons. If data are sufficient and adequate, we will indirectly compare the two tests in relation to their sensitivities or specificities. Test comparison will be performed by adding covariate's for different types of index tests into the model, and testing the significance (significance level $=0.05$ ) of the parameters of covariate's. Analyses will be performed using STATA and Review Manager.

\section{Investigations of heterogeneity}

We will investigate factors that may contribute to heterogeneous results in a subgroup analysis, such as healthcare setting (e.g. emergency room versus primary care), different health professionals (e.g. medical specialists versus nurses), age of patient population, and the influence of delayed verification. We will also perform a sensitivity analysis for study quality (i.e., QUADAS-2) to investigate methodological heterogeneity. We will use forest plots and sensitivities and specificities plotted using an HSROC curve for visual examination of heterogeneity between studies, and add covariates (i.e. settings, health professional, age, delayed verification, and QUADAS items) to investigate the heterogeneity between studies in the meta-analysis.

\section{Sensitivity analyses}

As reported above we will conduct both sensitivity and subgroup analyses to evaluate the impact of methodological quality, healthcare setting and healthcare professional, population (e.g. age group) and reference standard (e.g. 14 day proxy) has on the performance of the index tests. 


\section{REFERE N C E S}

\section{Additional references}

\section{ACEM / RANZCR Guidelines 2012}

Australasian College for Emergency Medicine (ACEM) and the Royal Australian and New Zealand College of Radiologists (RANZCR) Guidelines on diagnostic imaging 2012. www.acem.org.au/getattachment/17ee11f1-bdaf4ca4-a524-396690123ba7/Diagnostic-Imaging.aspx (accessed 25 Feb 2016).

\section{Ackland 2012}

Ackland H, Cameron P. Cervical spine - assessment following trauma. Australian Family Physician 2012;41(4): 196-201.

\section{ACR Appropriateness Criteria}

The American College of Radiology. ACR Appropriateness Criteria on suspected spine trauma. https://acsearch.acr.org/ docs/69359/Narrative/ (accessed 25 Feb 2016).

\section{Como 2009}

Como JJ, Diaz JJ, Dunham CM, Chiu WC, Duane TM, Capella JM, et al. Practice management guidelines for identification of cervical spine injuries following trauma: update from the eastern association for the surgery of trauma practice management guidelines committee. Journal of Trauma 2009;67(3):651-9.

\section{Daffner 2007}

Daffner RH, Hackney DB. ACR Appropriateness Criteria on suspected spine trauma. Journal of the American College of Radiology 2007;4(11):762-75.

Davis 1993

Davis JW, Phreaner DL, Hoyt DB, Mackersie RC. The etiology of missed cervical spine injuries. Journal of Trauma 1993;34:342-6.

Diagnostic Imaging Pathways

Diagnostic Imaging Pathways - Cervical Spine Injury. http://www.imagingpathways.health.wa.gov.au/index.php/ imaging-pathways/musculoskeletal-trauma/bone-and-jointtrauma/cervical-spine-injury\#pathway (accessed 25 Feb 2016).

\section{Goergen 2015}

Goergen S, Varma D, Ackland H, Michaleff Z, Rosenfeld JV, Malham G, et al. Adult Cervical Spine Trauma. Education Modules for Appropriate Imaging Referrals: Royal Australian and New Zealand College of Radiologists 2015.

Goode 2014

Goode T, Young A, Wilson SP, Katzen J, Wolfe LG, Duane TM. Evaluation of cervical spine fracture in the elderly: can we trust our physical examination?. American Surgeon 2014; 80(2):182-4.

Griffith 2011

Griffith B, Bolton C, Goyal N, Brown ML, Jain R. Screening cervical spine CT in a level I trauma center: overutilization?. AJR. American Journal of Roentgenology 2011;197(2):463-7.

\section{Griffith 2013}

Griffith B, Kelly M, Vallee P, Slezak M, Nagarwala J, Krupp $S$, et al. Screening cervical spine CT in the emergency department, Phase 2: A prospective assessment of use. AJNR. American Journal of Neuroradiology 2013;34(4): 899-903.

\section{Griffith 2014}

Griffith B, Vallee P, Krupp S, Jung M, Slezak M, Nagarwala J, et al. Screening cervical spine CT in the emergency department, phase 3: increasing effectiveness of imaging. Journal of the American College of Radiology 2014;11(2): $139-44$.

\section{Hasler 2012}

Hasler RM, Exadaktylos AK, Bouamra O, Benneker LM, Clancy M, Sieber R, et al. Epidemiology and predictors of cervical spine injury in adult major trauma patients: a multicenter cohort study. Journal of Trauma and Acute Care Surgery 2012;72(4):975-81.

\section{Hoffman 1998}

Hoffman JR, Wolfson AB, Todd K, Mower WR. Selective cervical spine radiography in blunt trauma: methodology of the National Emergency X-Radiography Utilization Study (NEXUS). Annals of Emergency Medicine 1998;32(4): 461-9.

\section{Hoffman 2000}

Hoffman JR, Mower WR, Wolfson AB, Todd KH, Zucker MI. Validity of a set of clinical criteria to rule out injury to the cervical spine in patients with blunt trauma. National Emergency X-Radiography Utilization Study Group. New England Journal of Medicine 2000;343:94-9.

\section{Holmes 2005}

Holmes JF, Akkinepalli R. Computed tomography versus plain radiography to screen for cervical spine injury: a metaanalysis. Journal of Trauma 2005;58(5):902-5.

\section{Matteucci 2015}

Matteucci MJ, Moszyk D, Migliore SA. Agreement between resident and faculty emergency physicians in the application of NEXUS criteria for suspected cervical spine injuries. Journal of Emergency Medicine 2015;48:445-9.

Milby 2008

Milby AH, Halpern CH, Guo W, Stein SC. Prevalence of cervical spinal injury in trauma. Neurosurgical Focus 2008; 25(5):E10.

\section{Morrison 2014}

Morrison J, Jeanmonod R. Imaging in the NEXUS-negative patient: when we break the rule. American Journal of Emergency Medicine 2014;32:67-70.

\section{Motor Accidents Authority 2014}

Motor Accidents Authority. Guidelines for the management of acute whiplash-associated disorders - for health professionals. Sydney: third edition. Sydney, 2014. 
NICE Guidance 2007

National Institute for Health and Care Excellence NICE guideline [CG176]. Triage, assessment, investigation and early management of head injury in infants, children and adults. National Institute for Health and Care Excellence September 2007.

\section{Niska 2010}

Niska R, Bhuiya F, Xu J. National Hospital Ambulatory Medical Care Survey: 2007 Emergency Department Summary. National Health Statistics Reports 2010;6(26): $1-31$.

\section{Parizel 2010}

Parizel PM, van der Zijden T, Gaudino S, Spaepen M, Voormolen MHJ, Venstermans C, et al. Trauma of the spine and spinal cord: imaging strategies. European Spine Journal 2010;19 (Suppl 1):S8-S17.

\section{Reitsma 2005}

Reitsma JB, Glas AS, Rutjes AW, Scholten RJ, Bossuyt $\mathrm{PM}, \mathrm{Zwinderman} \mathrm{AH}$. Bivariate analysis of sensitivity and specificity produces informative summary measures in diagnostic reviews. Journal of Clinical Epidemiology 2005; 58:982-90.

Slaar 2017

Slaar A, Fockens MM, Wang J, Maas M, Wilson DJ, Goslings JC, et al. Triage tools for detecting cervical spine injury in pediatric trauma patients. Cochrane Database of Systematic Reviews 2017, Issue 12. DOI: 10.1002/ 14651858.CD011686.pub2

\section{Stiell 1997}

Stiell IG, Wells GA, Vandemheen K, Laupacis A, Brison R, Eisenhauer MA, et al. Variation in emergency department use of cervical spine radiography for alert, stable trauma patients. CMAJ: Canadian Medical Association Journal 1997;156(11):1537-44.

\section{Stiell 1999}

Stiell IG, Lesiuk HJ, Vandemheen K, Worthington JR, Verbeek PR, Reardon M, et al. Obtaining Consensus for a Definition of "Clinically Important Cervical Spine Injury" in the CCC Study. Academic Emergency Medicine. 1999; Vol. 6:435.

\section{Stiell 2001}

Stiell IG, Wells GA, Vandemheen KL, Clement CM, Lesiuk H, De Maio VJ, et al. The Canadian C-spine rule for radiography in alert and stable trauma patients. JAMA 2001;286:1841-8.

Stiell 2003

Stiell IG, Clement CM, McKnight RD, Brison R, Schull $\mathrm{MJ}$, Rowe $\mathrm{BH}$, et al. The Canadian $\mathrm{C}$-spine rule versus the NEXUS low-risk criteria in patients with trauma. New England Journal of Medicine 2003;349:2510-8.

The College of Emergency Medicine 2010

The College of Emergency Medicine. Guideline on the management of alert, adult patients with potential cervical spine injury in the Emergency Department. HRH The Princess Royal, London November 2010.

TRACsa 2008

TRACsa: Trauma, Injury Recovery. Clinical guidelines for best practice management of acute and chronic whiplashassociated disorders. TRACsa, Adelaide:. Adelaide, 2008.

\section{Vandemheen 1999}

Vandemheen K, Stiell I G, Brison R, Cass D, Dreyer J, Eisenhauer MA, et al. Validity evaluation of the cervical spine injury proxy outcome assessment tool in the CCC study. Academic Emergency Medicine. 1999; Vol. 6: 434-5.

Wade 2013

Wade R, Corbett M, Eastwood A. Quality assessment of comparative diagnostic accuracy studies: our experience using a modified version of the QUADAS-2 tool. Research Synthesis Methods 2013;4(3):280-6.

Whiting 2011

Whiting PF, Rutjes AW, Westwood ME, Mallett S, Deeks JJ, Reitsma JB, et al. QUADAS-2 Group. QUADAS-2: a revised tool for the quality assessment of diagnostic accuracy studies. Annals of Internal Medicine 2011;155:529-36.

\section{References to other published versions of this review}

\author{
Michaleff 2012 \\ Michaleff ZA, Maher CG, Verhagen AP, Rebbeck T, Lin \\ CW. Accuracy of the Canadian C-spine rule and NEXUS \\ to screen for clinically important cervical spine injury \\ in patients following blunt trauma: a systematic review. \\ CMAJ: Canadian Medical Association Journal 2012;184 \\ E867-76. \\ * Indicates the major publication for the study
}

\section{ADDITIONAL TABLES}

\section{Table 1. The NEXUS Criteria}

\section{Criteria}

1. No posterior midline cervical tenderness
Explanations

Midline posterior bony cervical spine tenderness is present if the patient complains of pain on palpation of the posterior midline neck from the nuchal ridge to the prominence of the first thoracic vertebra, or if the patient reports pain with direct palpation of any 
Table 1. The NEXUS Criteria (Continued)

cervical spinous process

2. No evidence of intoxication

Patients should be considered intoxicated if they have either of the following: (a) a recent history, by the patient or an observed intoxication or intoxicating ingestion; or (b) evidence of intoxication on physical examination, such as odour of alcohol, slurred speech, ataxia, dysmetria, or other cerebellar findings, or any behavior consistent with intoxication. Patients may also be considered to be intoxicated if tests of bodily secretions are positive for drugs that affect level of alertness, including a blood alcohol level greater than $0.08 \mathrm{mg} / \mathrm{dL}$

3. Normal level of alertness

An altered level of alertness can include any of the following: (a) Glasgow Coma Scale score of 14 or less; (b) disorientation to person, place, time, or events; (c) inability to remember 3 objects at 5 minutes; (d) delayed or inappropriate response to external stimuli; or (e) other

4. No focal neurological deficit

Any focal neurological complaint (by history) or finding (on motor or sensory examination)

5. No painful distracting injuries

No precise definition for distracting painful injury is possible. This includes any condition thought by the clinician to be producing pain sufficient to distract the patient from a second (neck) injury. Examples may include, but are not limited to, the following: (a) a long bone fracture; (b) a visceral injury requiring surgical consultation; (c) a large laceration, degloving injury, or crush injury; (d) large burns; or (e) any other injury producing acute functional impairment. Physicians may also classify any injury as distracting if it is thought to have the potential to impair the patient's ability to appreciate other injuries

*If all of these criteria are met, imaging is not required in order to exclude clinically important CSI

Table 2. Assessment of methodological quality: modified version of QUADAS-2

\begin{tabular}{|c|c|c|c|c|}
\hline Quality domain & Risk of bias & Signalling questions & Applicability & Signalling questions \\
\hline 1: Patient selection & $\begin{array}{l}\text { Could the selection of } \\
\text { patients have introduced } \\
\text { bias? (high/low/unclear) }\end{array}$ & $\begin{array}{l}\text { 1) Was a consecutive or } \\
\text { random sample of pa- } \\
\text { tients enrolled? (yes/no/ } \\
\text { unclear) } \\
\text { 2) Did the study avoid } \\
\text { inappropriate exclu- } \\
\text { sions? (yes/no/unclear) }\end{array}$ & $\begin{array}{l}\text { Are there concerns that } \\
\text { the included patients and } \\
\text { settings do not match the } \\
\text { review question? (high/ } \\
\text { low/unclear) }\end{array}$ & $\begin{array}{l}\text { Were all the patients re- } \\
\text { cruited from the same } \\
\text { clinical setting? }\end{array}$ \\
\hline 2: Index test & $\begin{array}{l}\text { Could the interpretation } \\
\text { of the index test have } \\
\text { introduced bias? (high/ } \\
\text { low/unclear) }\end{array}$ & $\begin{array}{l}\text { 1) Were the index test re- } \\
\text { sults interpreted without } \\
\text { knowledge of the results } \\
\text { of the reference standard? } \\
\text { (yes/no/unclear) } \\
\text { 2) Did the whole sam- } \\
\text { ple or a random selec- }\end{array}$ & $\begin{array}{l}\text { Are there concerns that } \\
\text { the index test, its con- } \\
\text { duct, or the interpre- } \\
\text { tation differ from the } \\
\text { review question? (high/ } \\
\text { low/unclear) }\end{array}$ & $\begin{array}{l}\text { 1) Did the study pro- } \\
\text { vide a clear definition } \\
\text { of what was considered } \\
\text { to be a "positive" result } \\
\text { for the index test? (high/ } \\
\text { low/unclear) }\end{array}$ \\
\hline
\end{tabular}

Canadian C-spine rule and the National Emergency X-Radiography Utilization Study (NEXUS) for detecting clinically important 
Table 2. Assessment of methodological quality: modified version of QUADAS-2 (Continued)

tion of the sample receive verification using a reference standard? (yes/no/ unclear)
2) Was the index test implemented by the same type of health professional in all patients? (e. g. profession, level of training)
3: Reference Standard Could the interpretation of the reference standard have introduced bias? (high/low/unclear)
1) Is the reference standard likely to correctly classify the target condition?

2) Were the reference standard results interpreted without knowledge of the results of the index test?
4: Flow and timing

Could the patient flow have introduced bias? (low/high/unclear)
1) Was there an appropriate interval between index test and reference standard (e.g. short enough to be reasonably sure that the target condition did not change between the two tests)? 2) Did all patients receive the same reference standard?

3) Were all patients include in the analysis?

4) Were withdrawals from the study clearly reported?
Are there concerns that the target condition as defined by the preference standard does not match the review question? (high/low/unclear)
1) Did the study provide a clear definition of what was considered to be a "positive" result for the reference standard? 


\section{A P P E N D I C E S}

\section{Appendix I. Appendix I. MEDLINE search strategy}

1. (NEXUS or CCR).mp.

2. National Emergency X-Radiography.mp.

3. (Canadian c-spine or Canadian cervical spine).mp.

4. ((Cervical spine or c-spine) adj5 clear\$).mp.

5. (cervical adj5 (trauma $\$$ or injur $\$$ or fracture $\$$ or sublux $\$$ or dislocat $\$$ or avuls\$ or instab\$)).mp.

6. (Spinal cord injury without radiographic abnormality or SCIWORA).mp.

7. exp Cervical Vertebrae/

8. exp Neck Injuries/

9. exp Spinal Injuries/

10. exp Spinal Cord Injuries/

11. spinal fractures/

12. 1 or 2 or 3 or 4 [Index tests]

13. 5 or 6 or 7 or 8 or 9 or 10 or 11 [target condition]

14. 12 and 13

\section{WHAT'S NEW}

Date Event Description

8 May 2018 Amended Reference amended

\section{H I S T O R Y}

Protocol first published: Issue 4, 2018

\begin{tabular}{lll}
\hline Date & Event & Description \\
\hline 20 July 2015 & Amended & CMSG ID A114 \\
\hline
\end{tabular}

\section{CONTRIBUTIONSOFAUTHORS}

Conception, design and drafting of the protocol: Bruno T Saragiotto, Chris G Maher, Arianne P Verhagen, Stacy Georgen, and Zoe Michaleff.

Critical revision of the protocol for important intellectual content: Chris G Maher, Chung-Wei Christine Lin, Arianne P Verhagen, Stacy Goergen, Zoe A Michaleff

Final approval of the protocol: all authors. 


\section{DECLARATIONSOF INTEREST}

None known.

\section{SOURCES OF SUPPORT}

\section{Internal sources}

- National Health and Medical Research Council of Australia, Australia.

Prof Chris Maher is supported by the National Health and Medical Research Council of Australia (NHMRC) and Dr Christine Lin holds a career development fellowship from NHMRC.

\section{External sources}

- No sources of support supplied 